\title{
Weighted Gehring and Muckenhoupt classes and some in- clusion properties with norm inequalities
}

\author{
S. H. Saker, M. H. Hassan* \\ Department of Mathematics, Faculty of Science, Mansoura University, Mansoura 35516, Egypt.
}

\begin{abstract} by

In this paper, we will prove some fundamental properties of the power mean operator $\mathcal{H}_{\lambda} w^{p}$ of order $p$, which is defined

$$
\mathcal{H}_{\lambda} w^{p}(x)=\frac{1}{\Lambda(x)} \int_{0}^{x} \lambda(s) w^{p}(s) d s, \text { for } p \in \mathbb{R}^{+},
$$

where $\lambda$ and $w$ are nonnegative functions and $\Lambda(x)=\int_{0}^{x} \lambda(s)$ ds. Then by using these properties we will establish some norm inequalities of the generalized Muckenhoupt and Gehring weights and prove some fundamental relations between them.
\end{abstract}

Keywords: Hardy type inequality, generalized Muckenhoupt class, generalized Gehring class, inclusion properties.

2020 MSC: 26D10, 26D15.

(C)2022 All rights reserved.

\section{Introduction}

We fix an interval $I_{0} \subset \mathbb{R}$ and consider subintervals $I$ of $I_{0}$ and denote by $|\mathrm{I}|$ the Lebesgue measure of I. The weight $w$ is a nonnegative locally integrable function. The classical Hardy operator for a given weight $w$ reads as (see [15])

$$
\mathcal{H} w=\frac{1}{|\mathrm{I}|} \int_{\mathrm{I}} w(\mathrm{~s}) \mathrm{d} s
$$

for every subinterval I of $\mathrm{I}_{0}$. In terms of this operator the known $\mathcal{A}_{\mathrm{p}}(\mathrm{C})$-Muckenhoupt condition (see [28]) for a nonnegative measurable weight function $w$ can be expressed as

$$
(\mathcal{H} w)\left(\mathcal{H} w^{\frac{1}{1-p}}\right)^{p-1} \leqslant \mathcal{C},
$$

for $1<p<\infty$ and a constant $\mathcal{C}<\infty$. We say that the weight $w$ verifies the $\mathcal{A}_{1}$-Muckenhoupt condition if

$$
\mathcal{H} w \leqslant \mathcal{e} \inf _{\mathrm{I} \subset \mathrm{I}_{0}}(w)
$$

\footnotetext{
*Corresponding author

Email addresses: shsaker@mans .edu.eg (S. H. Saker), mohamed.hassan445@yahoo.com (M. H. Hassan)

doi: $10.22436 /$ jmcs.024.03.02
}

Received: 2020-12-13 Revised: 2021-01-01 Accepted: 2021-01-06 
for every subinterval I of $\mathrm{I}_{0}$. Coifman and Fefferman [5] proved that if $w$ satisfies $\mathcal{A}_{\mathrm{p}}$-Muckenhoupt condition (1.2), then there exists $\epsilon=\epsilon(p, \mathcal{C})>0$ such that

$$
(\mathcal{H} w)\left(\mathcal{H} w^{\frac{1}{1-q}}\right)^{q-1} \leqslant \mathcal{C}_{1}
$$

for all $p-\epsilon<q$, where the constant $\mathcal{C}_{1}=\mathcal{C}_{1}(\mathrm{p}, \mathcal{C})$. Muckenhoupt in [28] proved that if $w$ is nonincreasing and satisfies $\mathcal{A}_{1}$-Muckenhoupt condition (1.3), then there exists $p \in[1, \mathcal{C} /(\mathcal{C}-1)]$ such that

$$
\mathcal{H} w^{p} \leqslant \frac{\mathcal{C}}{\mathcal{C}-\mathrm{p}(\mathcal{C}-1)}(\mathcal{H} w)^{p} .
$$

Bojarski et al. [4] improved the Muckenhoupt inequality (1.4) by excluding the monotonicity condition and using rearrangement $w^{*}$ of the function $w$. In particular, they proved that if $w$ satisfies (1.3) with e $>1$, then

$$
\mathcal{H} w^{p} \leqslant \frac{\mathcal{C}^{1-p}}{\mathcal{C}-p(\mathcal{C}-1)}(\mathcal{H} w)^{p}, \text { for } p<\mathcal{C} /(\mathcal{C}-1)
$$

The constant on the right hand side of the inequality (1.5) as well as the upper bounds of $p$ cannot be improved. In [28] Muckenhoupt introduced the characterization of $\mathcal{A}_{p}$-condition in connection with the boundedness of the Hardy-Littlewood maximal operator

$$
\mathcal{M f}:=\sup _{\mathrm{I} \subset \mathrm{I}_{0}} \frac{1}{|\mathrm{I}|} \int_{\mathrm{I}} f(s) \mathrm{ds}
$$

in the space of $\mathrm{L}_{w}^{\mathrm{p}}\left(\mathrm{I}_{0}\right)$. In particular, Muckenhoupt proved that if $w$ is nonnegative weight and $1<p<\infty$, then there is a constant $K$ independent of $f$ for which

$$
\int_{I}(\mathcal{M} f)^{p} w(x) d x \leqslant K \int_{I}(f(x))^{p} w(x) d x
$$

holds if and only if there is a constant $\mathcal{C}$ independent of I for which (1.2) holds for all subintervals I of $\mathrm{I}_{0}$. In considering mean convergence problems for various series (see $[26,27,29])$ it was natural to consider the weighted Hardy-Littlewood maximal operator

$$
\mathcal{M} f(x):=\sup _{x \in I} \frac{1}{m(I)} \int_{I} f(t) d m(t),
$$

where $\mathrm{m}(\mathrm{I})$ is a suitable measure and the quotient is taken as zero if the numerator and the denominator are both zero or both infinity and $m(I)=\int_{I} d m(t)$. In [28] the author proved that if $m$ is a Borel measure on an interval $\mathrm{I}_{0}$, which is 0 on sets consisting of single points, and $1<p<\infty$, then $\mathcal{M} f$ is bounded, if and only if

$$
\left(\frac{1}{m(I)} \int_{I} w(t) d m(t)\right)\left(\frac{1}{m(I)} \int_{I} w^{-\frac{1}{p-1}}(t) d m(t)\right)^{p-1} \leqslant \mathcal{C},
$$

for every subinterval $\mathrm{I} \subset \mathrm{I}_{0}$, where $\mathcal{C}$ is a positive constant independent of $w$. This condition is a weighted Muckenhoupt condition in a measure space with a measure $m(I)$. For more related results, also tied to applications of functional inequalities toward the analysis of various problems, we refer the reader to the papers $[1-3,6-12,16,18-20,22-25,30-33]$ and the references cited therein.

Following this trend, by considering a generalized form of (1.1), we consider the power mean operator

$$
\mathcal{H}_{\lambda} w^{p}:=\frac{1}{\Lambda(I)} \int_{\mathrm{I}} \lambda(\mathrm{t}) w^{p}(\mathrm{t}) \mathrm{dt},
$$


where $\lambda$ and $w$ are nonnegative functions and $\Lambda(\mathrm{I})=\int_{\mathrm{I}} \lambda(\mathrm{t}) \mathrm{dt}$. Now, the corresponding $\mathcal{A}_{\mathrm{p}, \lambda}$-Muckenhoupt condition for $p>1$ and a nonnegative weight $w$ is given by

$$
\left(\mathcal{H}_{\lambda} w\right)\left(\mathcal{H}_{\lambda} w^{-\frac{1}{p-1}}\right)^{p-1} \leqslant \mathcal{C}, \text { for all } \mathrm{I} \subset \mathrm{I}_{0},
$$

for some constant $\mathcal{C}<\infty$. The smallest constant $\mathcal{C}$ satisfying (1.6) is called the $\mathcal{A}_{p, \lambda}$-norm and is denoted by

$$
\left[\mathcal{A}_{\mathrm{p}, \lambda}(w)\right]=\sup _{\mathrm{I} \subset \mathrm{I}_{0}} \mathcal{H}_{\lambda} w\left(\mathcal{H}_{\lambda} w^{-1 /(\mathrm{p}-1)}\right)^{\mathrm{p}-1}<\infty .
$$

For a given fixed constant $\mathcal{C}>1$ if $w$ is said to belong to $\mathcal{A}_{\mathrm{p}, \lambda}(\mathrm{C})$ then $\left[\mathcal{A}_{\mathrm{p}, \lambda}(w)\right] \leqslant \mathcal{C}$. The weight $w$ is said to belong to $\mathcal{A}_{1, \lambda}$, if there exists a constant $A \geqslant 1$, such that

$$
\mathcal{H}_{\lambda} w \leqslant A \inf _{t \in \mathrm{I}}(w), \text { for all } \mathrm{I} \subset \mathrm{I}_{0} .
$$

We define the $\mathcal{A}_{1, \lambda}$-norm by the following quantity

$$
\left[\mathcal{A}_{1, \lambda}(w)\right]=\sup _{\mathrm{I} \subset \mathrm{I}_{0}} \frac{\mathcal{H}_{\lambda} w}{\inf _{\mathrm{t} \in \mathrm{I}}(w(\mathrm{t}))}<\infty .
$$

The weight $w$ is said to belong to $\mathcal{A}_{\infty, \lambda}$, if

$$
\left[\mathcal{A}_{\infty, \lambda}(w)\right]=\sup _{\mathrm{I} \subset \mathrm{I}_{0}}\left(\mathcal{H}_{\lambda} w\right) \exp \left(\frac{1}{\Lambda(\mathrm{I})} \int_{\mathrm{I}} \lambda(\mathrm{s}) \log \frac{1}{w(\mathrm{~s})} \mathrm{ds}\right)<\infty .
$$

Another important class of weights which is related to the Muckenhoupt class is the $\mathrm{G}_{\mathrm{q}}$-class of weights for $1<\mathrm{q}<\infty$ that satisfy the reverse Hölder inequality. This class has been introduced by Gehring in $[13,14]$ in connection with local integrability properties of the gradient of quasiconformal mapping. The weight $w$ is said to belong to $G_{\mathrm{q}, \lambda}$ for $1<\mathrm{q}<\infty$, if there exists a constant $G \geqslant 1$, such that

$$
\left(\mathcal{H}_{\lambda} w^{\mathrm{q}}\right)^{1 / \mathrm{q}} \leqslant \mathrm{G} \mathcal{H}_{\lambda} w
$$

For $q>1$, we define $G_{q, \lambda}$-norm by

$$
\left[G_{q, \lambda}(w)\right]=\sup _{I \subset I_{0}}\left[\frac{\left(\mathcal{H}_{\lambda} w^{q}\right)^{\frac{1}{q}}}{\mathcal{H}_{\lambda} w}\right]^{q^{\prime}}<\infty
$$

where $q^{\prime}=q /(q-1)$. The function $w$ is said to belong to $G_{1, \lambda}$, if

$$
\left[\mathrm{G}_{1, \lambda}(w)\right]=\sup _{\mathrm{I} \subset \mathrm{I}_{0}}\left[\exp \left(\frac{1}{\Lambda(\mathrm{I})} \int_{\mathrm{I}} \frac{\lambda(\mathrm{x}) w(\mathrm{x})}{\mathcal{H}_{\lambda} w(x)} \log \left(\frac{w(x)}{\mathcal{H}_{\lambda} w(x)}\right) d x\right)\right]<\infty .
$$

The function $w$ is said to belong to $G_{\infty, \lambda}$, if

$$
\left[\mathrm{G}_{\infty, \lambda}(w)\right]=\sup _{\mathrm{I} \subset \mathrm{I}_{0}} \frac{w}{\mathcal{H}_{\lambda} w}<\infty
$$

For the inclusion properties between these two important classes, Bojarski et al. [4] (see (1.5)), showed that $\mathcal{A}_{1}(\mathcal{C}) \subset \mathrm{G}_{\mathrm{p}}(\mathcal{K})$ with

$$
\mathcal{K}=\left(\frac{\mathcal{C}^{1-p}}{\mathcal{C}-\mathrm{p}(\mathcal{C}-1)}\right)^{1 / p}, \text { and } \mathrm{p}<\mathcal{C} /(\mathcal{C}-1) .
$$


One way of establishing Gehring condition involves exploiting the correspondence between a weighted Muckenhoupt class and a reverse Hölder class. In fact from (1.7), we get that

$$
\left(\frac{1}{|I|} \int_{I} w^{q-\frac{1}{p-1}}(x)\left(\frac{1}{w(x)}\right)^{\frac{-1}{p-1}} d x\right)^{p-1} \leqslant \mathcal{K}^{q(p-1)}\left(\frac{1}{|I|} \int_{I} w(x) d x\right)^{q(p-1)} .
$$

By taking $q=p /(p-1)$, we have from (1.8) that (here $\left.\Lambda(I)=\int_{I} w(t) d t\right)$

$$
\begin{aligned}
& \left(\frac{1}{\Lambda(\mathrm{I})} \int_{\mathrm{I}} w(\mathrm{x})\left(\frac{1}{\mathcal{w}(\mathrm{x})}\right) \mathrm{d} x\right)\left(\frac{1}{\Lambda(\mathrm{I})} \int_{\mathrm{I}} w(x)\left(\frac{1}{w(x)}\right)^{\frac{-1}{\mathrm{p}-1}} \mathrm{~d} x\right)^{\mathrm{p}-1} \\
& \leqslant \mathcal{K}^{\mathrm{q}(\mathrm{p}-1)} \frac{|\mathrm{I}|^{\mathrm{p}}}{\Lambda^{\mathrm{p}(\mathrm{I})}}\left(\frac{\Lambda(\mathrm{I})}{|\mathrm{I}|}\right)^{\mathrm{p}}=\mathcal{K}^{\mathrm{p}}, \text { for all } \mathrm{I} \subset \mathrm{I}_{0}
\end{aligned}
$$

which is a weighted $\mathcal{A}_{\mathrm{p}, w}\left(\mathcal{K}^{\mathrm{p}}\right)$ condition for $w^{-1}$ with respect the weight $w$ and $\mathrm{p}=\mathrm{q} /(\mathrm{q}-1)$. This shows that if $w \in \mathrm{G}_{\mathrm{q}}(\mathcal{K})$, then $w^{-1} \in \mathcal{A}_{\mathrm{p}, w}(\mathrm{C})$ with $\mathrm{C}=\mathcal{K}^{1 / \mathrm{p}}$ where $\mathrm{p}=\mathrm{q} /(\mathrm{q}-1)$.

Following this subject, we will prove some fundamental properties of the generalized Muckenhoupt class $\mathcal{A}_{\mathrm{p}, \lambda}$ and Gehring class $\mathrm{G}_{\mathrm{q}, \lambda}$ with weights. The paper is organized as follows. In Section 2, we will prove some inequalities involving the generalized Hardy operator of order $p$, and also prove some inequalities with positive and negative powers. In Section 3, we will prove some inclusion properties of the Muckenhoupt weights as well as some norm inequalities. In Section 4, we will prove the same for the Gehring weights. In Section 5, we generalize the results to $\mathrm{G}_{\varphi}$-spaces.

\section{Some basic inequalities}

We fix an interval $\mathrm{I}_{0} \subset \mathbb{R}^{+}=[0, \infty)$ and consider subintervals $\mathrm{I}$ of $\mathrm{I}_{0}$ of the form $[0, \mathrm{~s}]$ for $0<\mathrm{s}<\infty$ (or $[0, \infty)$ ) and assume that $\lambda$ is a positive integrable function defined on $\mathrm{I}_{0}$. In the following, we state and prove some inequalities involving the generalized power mean operator

$$
\mathcal{H}_{\lambda} w^{p}(t)=\frac{1}{\Lambda(t)} \int_{0}^{t} \lambda(s) w^{p}(s) d s, \text { for } p \in \mathbb{R}^{+},
$$

where $\Lambda(t)=\int_{0}^{t} \lambda(s)$ ds. The proof of the following lemma is elementary and will be omitted.

Lemma 2.1. Assume that $w$ is any nonnegative weight. Then following properties hold:

(1) if $w$ is nonincreasing, then $\mathcal{H}_{\lambda} w$ is nonincreasing and $\mathcal{H}_{\lambda} w(t) \geqslant w(t)$, for all $\mathrm{t} \in \mathrm{I}$;

(2) if $w$ is nondecreasing, then $\mathcal{H}_{\lambda} w$ is nondecreasing and $\mathcal{H}_{\lambda} w(t) \leqslant w(t)$, for all $\mathrm{t} \in \mathrm{I}$.

The following lemmas are adapted from [15] and [17], respectively.

Theorem 2.2. Assume that $p>1$ and $\lambda$ and $w$ are nonnegative functions. Then

$$
\mathcal{H}_{\lambda}\left[\mathcal{H}_{\lambda} w(t)\right]^{p} \leqslant\left(\frac{p}{p-1}\right)^{p} \mathcal{H}_{\lambda} w^{p}(x)
$$

Theorem 2.3. Assume that $\lambda$ and $w$ are nonnegative functions. Then

$$
\mathcal{H}_{\lambda}\left[\exp \left(\mathcal{H}_{\lambda} \log w(t)\right)\right] \leqslant e \mathcal{H}_{\lambda} w(x) .
$$

Now, we prove an inequality of Hardy's type of negative powers.

Theorem 2.4. Assume that $\mathrm{p} \geqslant \mathrm{q}>0$ and $\lambda$ and $w$ are nonnegative functions such that $w$ is nonincreasing. Then

$$
\mathcal{H}_{\lambda}\left[\mathcal{H}_{\lambda} w(\mathrm{t})\right]^{-\mathrm{p}} \leqslant\left(\frac{\mathrm{p}+1}{\mathrm{p}}\right)^{\mathrm{q}} \mathcal{H}_{\lambda}\left[w^{-\mathrm{q}}(\mathrm{t})\left(\mathcal{H}_{\lambda} w(\mathrm{t})\right)^{-\mathrm{p}+\mathrm{q}}\right]
$$


Proof. Let

$$
y_{1}=\left(\frac{p}{p+1}\right)^{1+q / p} w^{q / p}(t)\left(\mathcal{H}_{\lambda} w(t)\right)^{1-q / p}, \quad \quad \text { and } y_{2}=\left(\frac{p}{p+1}\right)\left(\mathcal{H}_{\lambda} w(t)\right) .
$$

Thus

$$
y_{1}^{-p}=\left(\frac{p}{p+1}\right)^{-p-q} w^{-q}(t)\left(\mathcal{H}_{\lambda} w(t)\right)^{-p+q}, \quad y_{2}^{-p}=\left(\frac{p}{p+1}\right)^{-p}\left(\mathcal{H}_{\lambda} w(t)\right)^{-p}
$$

and

$$
\mathrm{y}_{1} \mathrm{y}_{2}^{-\mathrm{p}-1}=\left(\frac{\mathrm{p}}{\mathrm{p}+1}\right)^{-\mathrm{p}+\mathrm{q} / \mathrm{p}} w^{\mathrm{q} / \mathrm{p}}(\mathrm{t})\left(\mathcal{H}_{\lambda} w(\mathrm{t})\right)^{-\mathrm{p}-\mathrm{q} / \mathrm{p}}
$$

Since (see [15])

$$
y_{1}^{-p}+p y_{1} y_{2}^{-p-1}-(p+1) y_{2}^{-p} \geqslant 0,
$$

then we have that

$$
\begin{aligned}
(p+1)\left(\frac{p}{p+1}\right)^{-p}\left(\mathcal{H}_{\lambda} w(t)\right)^{-p} \leqslant & \left(\frac{p}{p+1}\right)^{-p-q} w^{-q}(t)\left(\mathcal{H}_{\lambda} w(t)\right)^{-p+q} \\
& +p\left(\frac{p}{p+1}\right)^{-p+q / p} w^{q / p}(t)\left(\mathcal{H}_{\lambda} w(t)\right)^{-p-q / p}
\end{aligned}
$$

So we get

$$
\begin{aligned}
(p+1)\left(\mathcal{H}_{\lambda} w(t)\right)^{-p} \leqslant & \left(\frac{p+1}{p}\right)^{q} w^{-q}(t)\left(\mathcal{H}_{\lambda} w(t)\right)^{-p+q} \\
& +p\left(\frac{p}{p+1}\right)^{q / p} w^{q / p}(t)\left(\mathcal{H}_{\lambda} w(t)\right)^{-p-q / p} .
\end{aligned}
$$

By integrating (2.4) from 0 to $x$, we have that

$$
\begin{aligned}
(p+1) \int_{0}^{x} \lambda(t)\left(\mathcal{H}_{\lambda} w(t)\right)^{-p} d t \leqslant & \left(\frac{p+1}{p}\right)^{q} \int_{0}^{x} \lambda(t) w^{-q}(t)\left(\mathcal{H}_{\lambda} w(t)\right)^{-p+q} d t \\
& +p\left(\frac{p+1}{p}\right)^{-q / p} \int_{0}^{x} \lambda(t) w^{q / p}(t)\left(\mathcal{H}_{\lambda} w(t)\right)^{-p-q / p} d t .
\end{aligned}
$$

Since $w$ is nonincreasing, we see that $w(t) \leqslant \mathcal{H}_{\lambda} w(t)$, and so

$$
w^{q / p}(t) \leqslant\left(\mathcal{H}_{\lambda} w(t)\right)^{q / p}, \text { for } \frac{q}{p}>1 \text {. }
$$

By combining the last inequality with (2.5), we obtain that

$$
\int_{0}^{x} \lambda(t) w^{q / p}(t)\left(\mathcal{H}_{\lambda} w(t)\right)^{-p-q / p} d t \leqslant \int_{0}^{x} \lambda(t)\left(\mathcal{H}_{\lambda} w(t)\right)^{-p} d t \leqslant\left(\frac{p+1}{p}\right)^{q / p} \int_{0}^{x} \lambda(t)\left(\mathcal{H}_{\lambda} w(t)\right)^{-p} d t .
$$

So we have that

$$
\frac{1}{\Lambda(x)} \int_{0}^{x} \lambda(t)\left(\mathcal{H}_{\lambda} w(t)\right)^{-p} d t \leqslant\left(\frac{p+1}{p}\right)^{q} \frac{1}{\Lambda(x)} \int_{0}^{x} \lambda(t) w^{-q}(t)\left(\mathcal{H}_{\lambda} w(t)\right)^{-p+q} d t
$$

that is,

$$
\mathcal{H}_{\lambda}\left[\mathcal{H}_{\lambda} w(\mathrm{t})\right]^{-\mathrm{p}} \leqslant\left(\frac{\mathrm{p}+1}{\mathrm{p}}\right)^{\mathrm{q}} \mathcal{H}_{\lambda}\left[w^{-\mathrm{q}}(\mathrm{t})\left(\mathcal{H}_{\lambda} w(\mathrm{t})\right)^{-\mathrm{p}+\mathrm{q}}\right],
$$

which is the desired inequality (2.3). The proof is complete. 
From Theorem 2.4, we get the following result (see also [2]).

Lemma 2.5. Assume that $\lambda$ and $w$ are nonnegative functions such that $w$ is nonincreasing. Then the following inequality

$$
\mathcal{H}_{\lambda}\left[\mathcal{H}_{\lambda} w(t)\right]^{-r} \mathrm{dt} \leqslant\left(\frac{r+1}{r}\right)^{r} \mathcal{H}_{\lambda} w^{-r}(x)
$$

holds for positive constants $r$.

\section{Norm inequalities on Muckenhoupt classes}

In this section, we will prove some inclusion properties of Muckenhoupt classes $\mathcal{A}_{p, \lambda}(w)$, and prove some norm inequalities with weights.

Theorem 3.1. Assume that $\lambda$ and $w$ are nonnegative functions such that $w$ is nondecreasing and $p>1$. Then $\left(\mathcal{H}_{\lambda} w(\mathrm{t})\right)^{\alpha} \in \mathcal{A}_{1, \lambda}$, if and only if $w \in \mathcal{A}_{\mathrm{p}, \lambda}$, and

$$
\left[\mathcal{A}_{p, \lambda}(w)\right]^{-\alpha} \leqslant \mathcal{A}_{1, \lambda}\left[\left(\mathcal{H}_{\lambda} w\right)^{\alpha}\right] \leqslant p^{-\alpha}\left[\mathcal{A}_{p, \lambda}(w)\right]^{-\alpha}
$$

where $\alpha=-1 /(p-1)$.

Proof. Assume that $w \in \mathcal{A}_{p, \lambda}$ and $\left[\mathcal{A}_{p, \lambda}(w)\right]=\mathcal{C}$. Then we get

$$
\sup _{x>0}\left[\mathcal{H}_{\lambda} w^{\alpha}(x)\right]^{p-1}\left(\mathcal{H}_{\lambda} w(x)\right) \leqslant \mathcal{C} .
$$

By applying (2.6) with $r=-\alpha=1 /(p-1)>0$, we see that

$$
\mathcal{H}_{\lambda}\left[\mathcal{H}_{\lambda} w(t)\right]^{\alpha} \leqslant\left(\frac{-\alpha+1}{-\alpha}\right)^{-\alpha}\left(\mathcal{H}_{\lambda} w^{\alpha}(x)\right)=p^{-\alpha}\left(\mathcal{H}_{\lambda} w^{\alpha}(x)\right) .
$$

Then

$$
\begin{aligned}
\sup _{x>0}\left[\mathcal{H}_{\lambda}\left(\mathcal{H}_{\lambda} w(t)\right)^{\alpha}\right]^{p-1}\left(\mathcal{H}_{\lambda} w(x)\right) & \leqslant\left(p^{-\alpha}\right)^{p-1} \sup _{x>0}\left[\mathcal{H}_{\lambda} w^{\alpha}(x)\right]^{p-1}\left(\mathcal{H}_{\lambda} w(x)\right) \\
& =\operatorname{psup}_{x>0}\left[\mathcal{H}_{\lambda} w^{\alpha}(x)\right]^{p-1}\left(\mathcal{H}_{\lambda} w(x)\right) \leqslant \mathcal{C p}
\end{aligned}
$$

Since that $-\alpha=1 /(p-1)>0$, so we have

$$
\sup _{x>0} \mathcal{H}_{\lambda}\left[\left(\mathcal{H}_{\lambda} w(t)\right)^{\alpha}\right]\left(\mathcal{H}_{\lambda} w(x)\right)^{\frac{1}{p-1}} \leqslant(\mathcal{C p})^{\frac{1}{p-1}}
$$

Thus

$$
\sup _{x>0} \frac{\mathcal{H}_{\lambda}\left[\left(\mathcal{H}_{\lambda} w(t)\right)^{\alpha}\right]}{\left[\mathcal{H}_{\lambda} w(x)\right]^{\alpha}} \leqslant(\mathcal{C p})^{-\alpha}
$$

Then we obtain

$$
A_{1, \lambda}\left[\left(\mathcal{H}_{\lambda} w(t)\right)^{\alpha}\right] \leqslant p^{-\alpha}\left[A_{p, \lambda}(w(t))\right]^{-\alpha},
$$

which is the right hand side in (3.1). Now, we assume that $\left(\mathcal{H}_{\lambda} w(t)\right)^{\alpha} \in A_{1, \lambda}$ and $A_{1, \lambda}\left[\left(\mathcal{H}_{\lambda} w(t)\right)^{\alpha}\right]=k$. Thus

$$
\sup _{x>0} \mathcal{H}_{\lambda}\left[\left(\mathcal{H}_{\lambda} w(t)\right)^{\alpha}\right] \leqslant k\left(\mathcal{H}_{\lambda} w(x)\right)^{\alpha}
$$

and then

$$
\sup _{x>0}\left[\mathcal{H}_{\lambda}\left(\mathcal{H}_{\lambda} w(t)\right)^{\alpha}\right]^{p-1} \leqslant k^{p-1}\left(\mathcal{H}_{\lambda} w(x)\right)^{-1}
$$


Since $w$ is nondecreasing then by Lemma 2.1, we have that $\mathcal{H}_{\lambda} w(t) \leqslant w(t)$ and then

$$
[w(t)]^{\frac{-1}{p-1}} \leqslant\left[\mathcal{H}_{\lambda} w(t)\right]^{\frac{-1}{p-1}} .
$$

So, since $\mathcal{H}_{\lambda} w$ is nondecreasing, that

$$
\mathcal{H}_{\lambda} w^{\alpha}(x) \leqslant \mathcal{H}_{\lambda}\left[\left(\mathcal{H}_{\lambda} w(t)\right)^{\alpha}\right] .
$$

Then, we have from (3.2), that

$$
\sup _{x>0}\left[\mathcal{H}_{\lambda} w^{\alpha}(x)\right]^{p-1} \leqslant \sup _{x>0}\left[\mathcal{H}_{\lambda}\left(\mathcal{H}_{\lambda} w(t)\right)^{\alpha}\right]^{p-1} \leqslant k^{p-1}\left(\mathcal{H}_{\lambda} w(x)\right)^{-1},
$$

and thus

$$
\sup _{x>0}\left(\mathcal{H}_{\lambda} w(x)\right)\left[\mathcal{H}_{\lambda} w^{\alpha}(x)\right]^{p-1} \leqslant k^{p-1},
$$

which gives that

$$
\left[\mathcal{A}_{\mathrm{p}, \lambda}(w)\right]^{-\alpha} \leqslant \mathcal{A}_{1, \lambda}\left[\left(\mathcal{H}_{\lambda}(w)\right)^{\alpha}\right],
$$

and thus we obtain the left hand side in (3.1). The proof is complete.

To illustrate the results in Theorem 3.1, we give the following example.

Example 3.2. Set $w_{\mathcal{\varepsilon}}(x)=\chi^{\varepsilon(p-1)}$ and $\lambda(x)=x^{(1-\gamma) p}$. By using the definition of the norms of $\mathcal{A}_{\mathrm{p}, \lambda}$ and $\mathcal{A}_{1, \lambda}$, we see that

$$
\left[\mathcal{A}_{p, \lambda}\left(w_{\varepsilon}\right)\right]=\frac{(1+p-p \gamma)^{p}}{(1+p-p \gamma-\varepsilon)^{p-1}[1+p-p \gamma+\varepsilon(p-1)]^{\prime}}
$$

and

$$
\mathcal{A}_{1, \lambda}\left[\left(\frac{1}{\Lambda(x)} \int_{0}^{x} \lambda(t) w_{\mathcal{\varepsilon}}(t) d t\right)^{-\frac{1}{p-1}}\right]=\frac{1+p-p \gamma}{1+p-p \gamma-\varepsilon}
$$

This implies that $w \in \mathcal{A}_{p, \lambda}$ and

$$
\left(\frac{1}{\Lambda(x)} \int_{0}^{x} \lambda(t) w_{\varepsilon}(t) d t\right)^{-\frac{1}{p-1}} \in \mathcal{A}_{1, \lambda}
$$

Then, we have

$$
\frac{\mathcal{A}_{1, \lambda}\left[\left(\frac{1}{\Lambda(x)} \int_{0}^{x} \lambda(t) w_{\varepsilon}(t) d t\right)^{-\frac{1}{p-1}}\right]}{\left[\mathcal{A}_{p, \lambda}\left(w_{\varepsilon}\right)\right]^{\frac{1}{p-1}}}=\frac{(1+p-p \gamma)[1+p-p \gamma+\varepsilon(p-1)]^{\frac{1}{p-1}}}{(1+p-p \gamma)^{\frac{p}{p-1}}} .
$$

As a special case when $\gamma=1$, then $\lambda(x)=1$ and $\Lambda(x)=x$. This implies that

$$
\mathcal{A}_{1}\left[\left(\frac{1}{x} \int_{0}^{x} w_{\varepsilon}(t) d t\right)^{-\frac{1}{p-1}}\right]=\frac{1}{1-\varepsilon}, \text { and }\left[\mathcal{A}_{p}\left(w_{\varepsilon}\right)\right]=\frac{(1-\varepsilon)^{-(p-1)}}{1+\varepsilon(p-1)},
$$

and thus

$$
\frac{\mathcal{A}_{1}\left[\left(\frac{1}{x} \int_{0}^{\chi} \mathcal{w}_{\mathcal{\varepsilon}}(\mathrm{t}) \mathrm{dt}\right)^{-\frac{1}{p-1}}\right]}{\left[\mathcal{A}_{\mathrm{p}}\left(\boldsymbol{w}_{\mathcal{\varepsilon}}\right)\right]^{\frac{1}{p-1}}}=[1+\varepsilon(\mathrm{p}-1)]^{\frac{1}{p^{-1}}} .
$$

By assuming that $\varepsilon=1$, we have that

$$
\mathcal{A}_{1}\left[\left(\frac{1}{x} \int_{0}^{x} w(\mathrm{t}) \mathrm{dt}\right)^{-\frac{1}{\mathrm{p}-1}}\right]=\mathrm{p}^{\frac{1}{\mathrm{p}-1}}\left[\mathcal{A}_{\mathrm{p}}(w)\right]^{\frac{1}{\mathrm{p}-1}},
$$

which is (3.1) with a sharp constant $p^{\frac{1}{p-1}}$. 
Theorem 3.3. Assume that $\lambda$ and $w$ are nonnegative functions such that $w$ is nonincreasing. Then, we have

$$
\mathcal{A}_{\infty, \lambda}(w) \leqslant \mathcal{A}_{1, \lambda}\left[\mathcal{H}_{\lambda} w(\mathrm{t})\right] \leqslant \mathrm{e} \mathcal{A}_{\infty, \lambda}(w) .
$$

Proof. Assume that $w \in \mathcal{A}_{\infty, \lambda}$ and there exists a positive constant $\mathcal{C}$ such that $\left[\mathcal{A}_{\infty, \lambda}(w)\right]=\mathcal{C}$, and

$$
\mathcal{H}_{\lambda} w(\mathrm{t}) \leqslant \mathcal{C} \exp \left(\mathcal{H}_{\lambda} \log w(\mathrm{t})\right) .
$$

So that

$$
\sup _{x>0} \mathcal{H}_{\lambda}\left[\mathcal{H}_{\lambda} w(t)\right] \leqslant \mathcal{C H} \mathcal{H}_{\lambda}\left[\exp \left(\mathcal{H}_{\lambda} \log w(t)\right)\right]
$$

By using (2.2), we get that

$$
\mathcal{H}_{\lambda}\left[\exp \left(\mathcal{H}_{\lambda} \log w(t)\right)\right] \leqslant e \mathcal{H}_{\lambda} w(x) .
$$

Then by using (3.4), and (2.2), we obtain

$$
\sup _{x>0} \mathcal{H}_{\lambda}\left[\mathcal{H}_{\lambda} w(t)\right] \leqslant \mathcal{e} \mathcal{H}_{\lambda}\left[\exp \left(\mathcal{H}_{\lambda} \log w(t)\right)\right] \leqslant \operatorname{Ce} \mathcal{H}_{\lambda} w(x)
$$

and thus

$$
\sup _{x>0} \mathcal{H}_{\lambda}\left[\mathcal{H}_{\lambda} w(t)\right] \leqslant \operatorname{Ce} \mathcal{H}_{\lambda} w(x)
$$

Then

$$
\sup _{x>0}\left[\frac{\mathcal{H}_{\lambda}\left[\mathcal{H}_{\lambda} w(t)\right]}{\mathcal{H}_{\lambda} w(x)}\right] \leqslant \mathcal{W},
$$

which is the right hand side in (3.3). Now, we assume that $\mathcal{H}_{\lambda} w(t) \in \mathcal{A}_{1, \lambda}$ that is $\mathcal{A}_{1, \lambda}\left[\mathcal{H}_{\lambda} w(t)\right]=k>0$. So, we have that

$$
\sup _{x>0} \mathcal{H}_{\lambda}\left[\mathcal{H}_{\lambda} w(t)\right] \leqslant k \mathcal{H}_{\lambda} w(x) .
$$

By the monotonicity of $\log w$, we get that

$$
\mathcal{H}_{\lambda} w(x) \leqslant \mathcal{H}_{\lambda}\left[\exp \left(\mathcal{H}_{\lambda} \log w(t)\right)\right]
$$

and thus

$$
\left.\sup _{x>0} \mathcal{H}_{\lambda}\left[\mathcal{H}_{\lambda} w(t)\right)\right] \leqslant k \mathcal{H}_{\lambda} w(x) \leqslant k \mathcal{H}_{\lambda}\left[\exp \left(\mathcal{H}_{\lambda} \log w(t)\right)\right]
$$

Then by using (3.5), we obtain that

$$
\sup _{x>0} \mathcal{H}_{\lambda} w(x) \leqslant k \exp \left(\mathcal{H}_{\lambda} \log w(x)\right),
$$

which implies that

$$
\sup _{x>0}\left[\mathcal{H}_{\lambda} w(x)\right] \exp \left(\mathcal{H}_{\lambda} \log \frac{1}{w(x)}\right) \leqslant k,
$$

and then $\left[\mathcal{A}_{\infty, \lambda}(w)\right] \leqslant k$, which is the left hand side in (3.3). The proof is complete.

To illustrate the result in Theorem 3.3, we give the following example.

Example 3.4. Assume that $w_{\varepsilon}(x)=x^{-\varepsilon}$ and $\lambda(x)=x^{-\alpha}$. So we have that

$$
\mathcal{A}_{\infty, \lambda}(w)=\frac{(1-\alpha)}{e^{\varepsilon /(1-\alpha)}(1-\varepsilon-\alpha)},
$$

and

$$
\mathcal{A}_{1, \lambda}\left[\left(\frac{1}{\Lambda(x)} \int_{0}^{x} \lambda(t) w_{\varepsilon}(t) d t\right)\right]=\frac{(1-\alpha)}{(1-\varepsilon-\alpha)}
$$


This implies that

$$
\frac{\mathcal{A}_{1, \lambda}\left[\left(\frac{1}{\Lambda(x)} \int_{0}^{x} \lambda(t) w_{\mathcal{E}}(t) d t\right)\right]}{\mathcal{A}_{\infty, \lambda}(w)}=e^{\varepsilon /(1-\alpha)} .
$$

If $\alpha=0$, then $\lambda(x)=1$ and $\Lambda(x)=x$, and then we have that

$$
\mathcal{A}_{\infty}(w)=\frac{1}{e^{\varepsilon}(1-\varepsilon)}, \text { and } \mathcal{A}_{1}\left[\left(\frac{1}{x} \int_{0}^{x} w_{\varepsilon}(t) d t\right)\right]=\frac{1}{1-\varepsilon},
$$

and thus

$$
\mathcal{A}_{1}\left[\left(\frac{1}{x} \int_{0}^{x} w_{\varepsilon}(t) d t\right)\right]=e^{\varepsilon} \mathcal{A}_{\infty}(w) .
$$

If we put $\varepsilon=1$, we get the (3.3) with a sharp constant $e$.

The following inequality generates nonincreasing $\mathcal{A}_{1}$ - functions related to the generalized power mean operator $\mathcal{H}_{\lambda} w(t)$ as follows.

Theorem 3.5. Assume that $\lambda$ and $w$ are nonnegative functions such that $w$ is nonincreasing. If $0<\delta<1$, then

$$
\mathcal{H}_{\lambda}\left[\mathcal{H}_{\lambda} w(t)\right]^{\delta} \leqslant \frac{1}{1-\delta}\left[\mathcal{H}_{\lambda} w(x)\right]^{\delta} .
$$

Proof. By applying Fubini Theorem, we have that

$$
\begin{aligned}
\int_{0}^{x}\left(\frac{\lambda(t)}{\Lambda^{\delta}(t)} \int_{0}^{t} \lambda(s) w(s) d s\right) d t & =\int_{0}^{x} \lambda(t) w(t)\left(\int_{t}^{x} \frac{\lambda(s)}{\Lambda^{\delta}(s)} d s\right) d t \\
& =\left(\int_{0}^{x} \lambda(t) w(t) d t\right)\left[\frac{\Lambda^{(1-\delta)}(x)}{(1-\delta)}-\frac{\Lambda^{(1-\delta)}(t)}{(1-\delta)}\right] \\
& \leqslant \frac{\Lambda^{(1-\delta)}(x)}{(1-\delta)}\left(\int_{0}^{x} \lambda(t) w(t) d t\right)
\end{aligned}
$$

Then we see that

$$
\int_{0}^{x}\left(\frac{\lambda(t)}{\Lambda^{\delta}(t)} \int_{0}^{t} \lambda(s) w(s) d s\right) d t \leqslant \frac{\Lambda^{(1-\delta)}(x)}{(1-\delta)} \int_{0}^{x} \lambda(t) w(t) d t
$$

By applying the Hölder inequality and using (3.7), we obtain that

$$
\begin{aligned}
\int_{0}^{x} \lambda(t)\left(\mathcal{H}_{\lambda} w(t)\right)^{\delta} d t & =\int_{0}^{x} \frac{\lambda^{(1-\delta)}(t) \lambda^{\delta}(t)}{\Lambda^{\delta(1-\delta)}(t)}\left(\frac{1}{\Lambda^{\delta}(t)} \int_{0}^{t} \lambda(s) w(s) d s\right)^{\delta} d t \\
& \leqslant\left[\int_{0}^{x} \frac{\lambda(t)}{\Lambda^{\delta}(t)} d t\right]^{1-\delta}\left[\int_{0}^{x}\left(\frac{\lambda(t)}{\Lambda^{\delta}(t)} \int_{0}^{t} \lambda(s) w(s) d s\right) d t\right]^{\delta} \\
& =\left[\frac{\Lambda^{(1-\delta)}(x)}{(1-\delta)}\right]^{1-\delta}\left[\int_{0}^{x}\left(\frac{\lambda(t)}{\Lambda^{\delta}(t)} \int_{0}^{t} \lambda(s) w(s) d s\right) d t\right]^{\delta} \\
& \leqslant \frac{\Lambda^{(1-\delta)^{2}}(x)}{(1-\delta)^{(1-\delta)}}\left[\frac{\Lambda^{(1-\delta)}(x)}{(1-\delta)}\left(\int_{0}^{x} \lambda(t) w(t) d t\right)\right]^{\delta} \\
& =\frac{\Lambda^{(1-\delta)}(x)}{(1-\delta)}\left(\int_{0}^{x} \lambda(t) w(t) d t\right)^{\delta}
\end{aligned}
$$

This implies that

$$
\frac{1}{\Lambda(x)} \int_{0}^{x} \lambda(t)\left(\mathcal{H}_{\lambda} w(t)\right)^{\delta} d t \leqslant \frac{1}{(1-\delta)}\left(\frac{1}{\Lambda(x)} \int_{0}^{x} \lambda(t) w(t) d t\right)^{\delta}
$$


Then from (3.8), we have

$$
\mathcal{H}_{\lambda}\left[\mathcal{H}_{\lambda} w(t)\right]^{\delta} \leqslant \frac{1}{(1-\delta)}\left[\mathcal{H}_{\lambda} w(x)\right]^{\delta},
$$

which is desired inequality (3.6). The proof is complete.

We conclude this section by considering another inequality which follows from Theorem 3.1 and gives us explicit examples of nondecreasing $\mathcal{A}_{\mathfrak{p}, \lambda}$-functions in a similar way as Theorem 3.5 gives us nonincreasing $\mathcal{A}_{1, \lambda}$-functions.

Theorem 3.6. Assume that $\lambda$ and $v$ are nonnegative functions such that $v$ is nonincreasing. If $p>1$, and $0<\theta<1$, then

$$
\mathcal{A}_{p, \lambda}\left[\left(\mathcal{H}_{\lambda} v\right)^{-\theta}\right] \leqslant\left(\frac{p-1}{p-1-\theta}\right)^{p-1} .
$$

Proof. Since $v$ is nonincreasing, then we see that $\mathcal{H}_{\lambda} v(t)$ is also nonincreasing. So by setting $w(t)=$ $\left(\mathcal{H}_{\lambda} v(t)\right)^{-\theta}$, we see that $w$ is nondecreasing. Then for $\alpha=-1 /(p-1)$, we get that

$$
\mathcal{H}_{\lambda} w(t) \leqslant w(t)=\left[(w(t))^{-\alpha}\right]^{p-1},
$$

also we have

$$
\left[\mathcal{H}_{\lambda} w(t)\right]\left[\mathcal{H}_{\lambda} w^{\alpha}(t)\right]^{p-1} \leqslant\left[\left[\mathcal{H}_{\lambda} w^{\alpha}(t)\right]((w(t)))^{-\alpha}\right]^{p-1}=\left[\mathcal{H}_{\lambda} w^{\alpha}(t)[w(t)]^{\frac{1}{p-1}}\right]^{p-1} .
$$

Since $w(t)=\left(\mathcal{H}_{\lambda} v(t)\right)^{-\theta}$, we have from (3.10) that

$$
\begin{aligned}
\sup _{x>0} \mathcal{H}_{\lambda}\left[\left(\mathcal{H}_{\lambda} v(t)\right)^{-\theta}\right]\left[\mathcal{H}_{\lambda}\left[\left(\mathcal{H}_{\lambda} v(t)\right)^{\frac{\theta}{p-1}}\right]\right]^{p-1} & \leqslant \sup _{x>0}\left[\frac{\mathcal{H}_{\lambda}\left[\left(\mathcal{H}_{\lambda} v(t)\right)^{-\alpha \theta}\right]}{\left(\mathcal{H}_{\lambda} v(x)\right)^{-\alpha \theta}}\right] \\
& =\sup _{x>0}\left[\mathcal{H}_{\lambda}\left[\left(\mathcal{H}_{\lambda} v(t)\right)^{-\alpha \theta}\right]\left(\mathcal{H}_{\lambda} v(x)\right)^{\alpha \theta}\right]^{p-1},
\end{aligned}
$$

and so

$$
\mathcal{A}_{p, \lambda}\left[\left(\mathcal{H}_{\lambda} v\right)^{-\theta}\right] \leqslant\left[\mathcal{A}_{1, \lambda}\left(\left(\mathcal{H}_{\lambda} v\right)^{-\alpha \theta}\right)\right]^{p-1} .
$$

Then by applying (3.6) for $-\alpha \theta$, and letting $\delta=-\alpha \theta=\theta /(p-1)<1$, we see that

$$
\mathcal{A}_{1, \lambda}\left[\left(\mathcal{H}_{\lambda} \mathcal{v}\right)^{-\alpha \theta}\right] \leqslant \frac{1}{1-\delta}=\frac{1}{1+\alpha \theta}
$$

so we obtain

$$
\mathcal{A}_{p, \lambda}\left[\left(\mathcal{H}_{\lambda} v\right)^{-\theta}\right] \leqslant \mathcal{A}_{1, \lambda}\left[\left(\mathcal{H}_{\lambda} v\right)^{-\alpha \theta}\right]^{p-1} \leqslant \frac{1}{1+\alpha \theta}=\left(\frac{p-1}{p-1-\theta}\right)^{p-1},
$$

which is desired inequality (3.9). The proof is complete.

\section{Norm inequalities on Gehring classes}

In this section, we state some properties of weighted Gehring class $G_{q, \lambda}(w)$, and begin with the connection between Muckenhoupt $\mathcal{A}_{1, \lambda}$-class and $\mathrm{G}_{\mathrm{q}, \lambda}$-class.

Theorem 4.1. Assume that $\lambda$ and $w$ are nonnegative functions such that $w$ is nonincreasing, and $\mathrm{q}>1$. Then $\mathcal{H}_{\lambda}^{\mathrm{q}} w(\mathrm{t}) \in \mathcal{A}_{1, \lambda}$ if and only if $w \in \mathrm{G}_{\mathrm{q}, \lambda}$, and

$$
\left[\mathrm{G}_{\mathrm{q}, \lambda}(w)\right]^{\frac{1}{\mathrm{q}^{\prime}}} \leqslant\left[\mathcal{A}_{1, \lambda}\left(\mathcal{H}_{\lambda}^{\mathrm{q}} w\right)\right]^{\frac{1}{\mathrm{q}}} \leqslant \frac{\mathrm{q}}{\mathrm{q}-1}\left[\mathrm{G}_{\mathrm{q}, \lambda}(w)\right]^{\frac{1}{\mathrm{q}^{\prime}}},
$$

where $\mathrm{q}^{\prime}=\mathrm{q} /(\mathrm{q}-1)$. 
Proof. Assume that $\left[\mathrm{G}_{\mathrm{q}, \lambda}(w)\right]=\mathrm{e}^{\mathrm{q}^{\prime} / \mathrm{q}}$. Then

$$
\sup _{x>0} \mathcal{H}_{\lambda} w^{\mathrm{q}}(x) \leqslant \mathcal{C}\left[\mathcal{H}_{\lambda} w(x)\right]^{\mathrm{q}} \text {. }
$$

By using (2.1), for $q>1$, then we have that

$$
\sup _{x>0} \mathcal{H}_{\lambda}\left[\mathcal{H}_{\lambda} w(t)\right]^{\mathrm{q}} \leqslant\left(\frac{\mathrm{q}}{\mathrm{q}-1}\right)^{\mathrm{q}} \sup _{x>0} \mathcal{H}_{\lambda} w^{\mathrm{q}}(x) \leqslant \mathcal{C}\left(\frac{\mathrm{q}}{\mathrm{q}-1}\right)^{\mathrm{q}}\left[\mathcal{H}_{\lambda} w(x)\right]^{\mathrm{q}},
$$

thus

$$
\sup _{x>0}\left[\frac{\mathcal{H}_{\lambda}\left[\mathcal{H}_{\lambda} w(t)\right]^{q}}{\left[\mathcal{H}_{\lambda} w(x)\right]^{q}}\right] \leqslant \mathcal{C}\left(\frac{q}{q-1}\right)^{q}
$$

Then we obtain

$$
\left[\mathcal{A}_{1, \lambda}\left(\mathcal{H}_{\lambda} w(\mathrm{t})\right)^{\mathrm{q}}\right]^{\frac{1}{q}} \leqslant \mathrm{e}^{\frac{1}{q}}\left(\frac{\mathrm{q}}{\mathrm{q}-1}\right),
$$

which is the right hand side in (4.1). Let $\left(\mathcal{H}_{\lambda} w(t)\right)^{q} \in \mathcal{A}_{1, \lambda}(w)$, and $\mathcal{A}_{1, \lambda}\left[\left(\mathcal{H}_{\lambda} w(t)\right)^{q}\right]=k$. Then

$$
\sup _{x>0} \mathcal{H}_{\lambda}\left[\left(\mathcal{H}_{\lambda} w(t)\right)^{\mathrm{q}}\right] \leqslant k\left[\mathcal{H}_{\lambda} w(x)\right]^{\mathrm{q}} .
$$

Since $w$ is nonincreasing, then $\mathcal{H}_{\lambda} w^{\mathrm{q}}(x) \leqslant \mathcal{H}_{\lambda}\left[\left(\mathcal{H}_{\lambda} w(\mathrm{t})\right)^{\mathrm{q}}\right]$. So by using (4.2), we obtain that

$$
\sup _{x>0} \mathcal{H}_{\lambda} w^{\mathrm{q}}(x) \leqslant \sup _{\chi>0} \mathcal{H}_{\lambda}\left[\left(\mathcal{H}_{\lambda} w(\mathrm{t})\right)^{\mathrm{q}}\right] \leqslant k\left[\mathcal{H}_{\lambda} w(x)\right]^{\mathrm{q}}
$$

and thus

$$
\sup _{x>0} \mathcal{H}_{\lambda} w^{\mathrm{q}}(x) \leqslant k\left[\mathcal{H}_{\lambda} w(x)\right]^{\mathrm{q}}
$$

So that

$$
\left[G_{q, \lambda}(w)\right]^{\frac{1}{q^{\prime}}}=\sup _{x>0}\left[\frac{\left[\mathcal{H}_{\lambda} w^{q}(x)\right]^{\frac{1}{q}}}{\mathcal{H}_{\lambda} w(x)}\right] \leqslant k^{\frac{1}{q}},
$$

which is the left hand side in (4.1). The proof is complete.

To illustrate the results in Theorem 4.1, we give the following example.

Example 4.2. Assume that $w_{\mathcal{E}}(x)=x^{(\epsilon-1) / q}$ and $\lambda(x)=x^{(1-\alpha) / q}$. Then, we get

$$
\left[\mathrm{G}_{\mathrm{q}, \lambda}\left(w_{\varepsilon}\right)\right]^{\frac{1}{\mathrm{q}^{\prime}}}=\left(\frac{\mathrm{q}-\alpha+1}{1-\alpha+\mathrm{q} \varepsilon}\right)^{\frac{1}{\mathrm{q}}}\left(\frac{\mathrm{q}-\alpha+\varepsilon}{\mathrm{q}-\alpha+1}\right),
$$

and

$$
\left[\mathcal{A}_{1, \lambda}\left(\frac{1}{\Lambda(x)} \int_{0}^{x} \lambda(t) w_{\mathcal{\varepsilon}}(t) d t\right)^{q}\right]^{\frac{1}{q}}=\left(\frac{q-\alpha+1}{1-\alpha+q \varepsilon}\right)^{\frac{1}{q}}
$$

Thus

$$
\frac{\left[\mathcal{A}_{1, \lambda}\left(\frac{1}{\Lambda(x)} \int_{0}^{x} \lambda(t) w_{\varepsilon}(t) d t\right)^{q}\right]^{\frac{1}{q}}}{\left[G_{q, \lambda}\left(w_{\varepsilon}\right)\right]^{\frac{1}{q^{\prime}}}}=\frac{q-\alpha+1}{q-\alpha+\epsilon} .
$$

If $\alpha=1, \lambda(x)=1$, then $\wedge(x)=x$, and so we have that

$$
\left[\mathrm{G}_{\mathrm{q}}\left(w_{\varepsilon}\right)\right]^{\frac{1}{q^{\prime}}}=\left(\frac{1}{\varepsilon}\right)^{\frac{1}{q}}\left(\frac{\mathrm{q}-1+\varepsilon}{\mathrm{q}}\right),
$$


and

$$
\left[\mathcal{A}_{1}\left(\frac{1}{x} \int_{0}^{x} w_{\varepsilon}(t) d t\right)^{q}\right]^{\frac{1}{q}}=\left(\frac{1}{\varepsilon}\right)^{\frac{1}{q}} .
$$

This implies that

$$
\left[\mathcal{A}_{1}\left(\frac{1}{x} \int_{0}^{x} w_{\varepsilon}(t) d t\right)^{\mathrm{q}}\right]^{\frac{1}{q}}=\frac{\mathrm{q}}{\mathrm{q}+\epsilon-1}\left[\mathrm{G}_{\mathrm{q}}\left(w_{\varepsilon}\right)\right]^{\frac{1}{\mathrm{q}^{\prime}}} .
$$

Now, if we put $\varepsilon=0$, we obtain that

$$
\frac{\left[\mathcal{A}_{1}\left(\frac{1}{x} \int_{0}^{x} w(t) d t\right)^{q}\right]^{\frac{1}{q}}}{\left[G_{q}(w)\right]^{\frac{1}{q^{\prime}}}}=\frac{q}{q-1},
$$

a sharp constant $\mathrm{q} /(\mathrm{q}-1)$.

\section{Norm inequalities on $\mathbf{G}_{\varphi}$-spaces}

In this section, we consider a more general Gehring class by providing a set of nonnegative and convex functions $\varphi$, with second order derivative $\varphi^{\prime \prime}$ positive in $(0,+\infty)$ which satisfy the following Levinson condition

$$
\exists p>1: \varphi \cdot \varphi^{\prime \prime} \geqslant\left(1-\frac{1}{p}\right)\left(\varphi^{\prime}\right)^{2}
$$

It is obvious that the function $\varphi(t)=t^{p}$, for $p>1$, and fulfills this condition.

Definition 5.1. For a positive convex function $\varphi$, we say that the nonincreasing function $w$ is said to belong to $\mathrm{G}_{\varphi, \lambda}$, if

$$
\left[\mathrm{G}_{\varphi, \lambda}(w)\right]=\sup _{\mathrm{t}>0} \frac{\mathcal{H}_{\lambda} \varphi(w(t))}{\varphi\left(\mathcal{H}_{\lambda} w(t)\right)}
$$

where

$$
\varphi\left(\mathcal{H}_{\lambda} w(t)\right)=\varphi\left(\frac{1}{\Lambda(t)} \int_{0}^{t} \lambda(s) w(s) d s\right)
$$

and

$$
\mathcal{H}_{\lambda} \varphi(w(x))=\frac{1}{\Lambda(x)} \int_{0}^{x} \lambda(t) \varphi(w(t)) d t
$$

Theorem 5.2 ([21, Theorem 2]). Assume that $w$ is nonincreasing function and the condition (5.1) holds for any positive convex function $\varphi$. If $\mathrm{p}>1$, then

$$
\mathcal{H}_{\lambda}\left[\varphi\left(\mathcal{H}_{\lambda} w(t)\right)\right] \leqslant\left(\frac{p}{p-1}\right)^{p} \mathcal{H}_{\lambda} \varphi(w(x)) .
$$

Theorem 5.3. Assume that $w$ is nonincreasing function and the condition (5.1) holds for any positive nondecreasing convex function $\varphi$. If $\mathrm{p}>1$, then $\varphi\left(\mathcal{H}_{\lambda} w(\mathrm{t})\right) \in \mathcal{A}_{1, \lambda}$, if and only if $w \in \mathrm{G}_{\varphi, \lambda}$, and

$$
\left[G_{\varphi, \lambda}(w)\right] \leqslant \mathcal{A}_{1, \lambda}\left[\varphi\left(\mathcal{H}_{\lambda} w\right)\right] \leqslant\left(\frac{p}{p-1}\right)^{p}\left[G_{\varphi, \lambda}(w)\right]
$$

Proof. Let $w$ belongs to $\mathrm{G}_{\varphi, \lambda}$ and $\left[\mathrm{G}_{\varphi, \lambda}(w)\right]=\mathcal{C}$. Then

$$
\sup _{x>0} \mathcal{H}_{\lambda} \varphi(w(x)) \leqslant \mathcal{C} \varphi\left(\mathcal{H}_{\lambda} w(x)\right)
$$


By using (5.2), we obtain

$$
\sup _{x>0} \mathcal{H}_{\lambda}\left[\varphi\left(\mathcal{H}_{\lambda} w(t)\right)\right] \leqslant \sup _{x>0}\left(\frac{p}{p-1}\right)^{p} \mathcal{H}_{\lambda} \varphi(w(x)) .
$$

So we see that

$$
\sup _{\chi>0} \mathcal{H}_{\lambda}\left[\varphi\left(\mathcal{H}_{\lambda} w(t)\right)\right] \leqslant \mathcal{C}\left(\frac{p}{p-1}\right)^{p} \varphi\left(\mathcal{H}_{\lambda} w(x)\right)
$$

so that

$$
A_{1, \lambda}\left[\varphi\left(\mathcal{H}_{\lambda} w(t)\right)\right]=\sup _{x>0} \frac{\mathcal{H}_{\lambda}\left[\varphi\left(\mathcal{H}_{\lambda} w(t)\right)\right]}{\varphi\left(\mathcal{H}_{\lambda} w(x)\right)} \leqslant \mathcal{C}\left(\frac{p}{p-1}\right)^{p},
$$

which is the right hand side in (5.3). Let $\varphi\left(\mathcal{H}_{\lambda} w(t)\right) \in \mathcal{A}_{1, \lambda}$, and $\mathcal{A}_{1, \lambda}\left[\varphi\left(\mathcal{H}_{\lambda} w(t)\right)\right]=k$. Thus

$$
\sup _{x>0} \mathcal{H}_{\lambda}\left[\varphi\left(\mathcal{H}_{\lambda} w(t)\right)\right] \leqslant k \varphi\left(\mathcal{H}_{\lambda} w(x)\right) .
$$

By the monotonicity of $w$ and $\varphi$, then

$$
\sup _{x>0} \mathcal{H}_{\lambda} \varphi(w(x)) \leqslant \sup _{x>0} \mathcal{H}_{\lambda}\left[\varphi\left(\mathcal{H}_{\lambda} w(t)\right)\right] \leqslant k \varphi\left(\mathcal{H}_{\lambda} w(x)\right),
$$

so

$$
\left[G_{\varphi, \lambda}(w)\right] \leqslant k,
$$

which is the left hand side of in (5.3). The proof is complete.

Theorem 5.4. Assume that $w$ is nonincreasing function and the condition (5.1) holds for any positive nondecreasing convex function $\varphi$. Then $w \in \mathcal{A}_{\infty, \lambda}$ if and only if $\varphi^{-1}(w) \in \mathrm{G}_{\varphi, \lambda}$.

Proof. Let $\varphi^{-1}(w) \in \mathrm{G}_{\varphi, \lambda}$ and

$$
\left[\mathrm{G}_{\varphi, \lambda}\left[\varphi^{-1}(w)\right]\right]=\sup _{x>0} \frac{\mathcal{H}_{\lambda} w(x)}{\varphi\left[\mathcal{H}_{\lambda} \varphi^{-1}(w(x))\right]}=k .
$$

So we have

$$
\sup _{x>0} \mathcal{H}_{\lambda} w(x) \leqslant k \varphi\left[\mathcal{H}_{\lambda} \varphi^{-1}(w(x))\right]
$$

Since that $\varphi=\exp , \varphi^{-1}=\log$, then we get

$$
\sup _{x>0} \mathcal{H}_{\lambda} w(x) \leqslant k \exp \left[\mathcal{H}_{\lambda} \log (w(x))\right]
$$

So we obtain

$$
A_{\infty, \lambda}(w)=\sup _{x>0} \frac{\mathcal{H}_{\lambda} w(x)}{\exp \left[\mathcal{H}_{\lambda} \log (w(x))\right]} \leqslant k .
$$

Let that $w \in \mathcal{A}_{\infty, \lambda}$ and $\left[\mathcal{A}_{\infty, \lambda}(w)\right]=\mathcal{C}$. Then

$$
\sup _{x>0} \mathcal{H}_{\lambda} w(x) \leqslant \mathcal{C} \exp \left[\mathcal{H}_{\lambda} \log (w(x))\right] .
$$

Since that $\varphi=\exp , \varphi^{-1}=\log$, we obtain that

$$
\sup _{x>0} \mathcal{H}_{\lambda} w(x) \leqslant \mathcal{e} \varphi\left[\mathcal{H}_{\lambda} \varphi^{-1}(w(x))\right] .
$$

Since $w(x)=\varphi\left[\varphi^{-1}(w(x))\right]$, so we have

$$
\left[G_{\varphi, \lambda}\left[\varphi^{-1}(w)\right]\right]=\sup _{x>0} \frac{\mathcal{H}_{\lambda}\left[\varphi\left(\varphi^{-1} w(x)\right)\right]}{\varphi\left(\mathcal{H}_{\lambda} \varphi^{-1}(w(x))\right)}=\sup _{x>0} \frac{\mathcal{H}_{\lambda} w(x)}{\varphi\left[\mathcal{H}_{\lambda} \varphi^{-1}(w(x))\right]} \leqslant \mathcal{C} .
$$

The proof is complete. 
Theorem 5.5. Assume that $w$ is nonincreasing function and the condition (5.1) holds for any positive nondecreasing convex function $\varphi$. Then $\mathcal{H}_{\lambda} w(\mathrm{t}) \in \mathcal{A}_{1, \lambda}$, if and only if $\varphi\left[\mathcal{H}_{\lambda} \varphi^{-1}(w(\mathrm{t}))\right] \in \mathcal{A}_{1, \lambda}$.

Proof. Let $\mathcal{H}_{\lambda} w(\mathrm{t}) \in \mathcal{A}_{1, \lambda}$, and $\mathcal{A}_{1, \lambda}\left[\mathcal{H}_{\lambda} w(\mathrm{t})\right]=k$. Then we get that

$$
\sup _{x>0} \mathcal{H}_{\lambda}\left[\mathcal{H}_{\lambda} w(t)\right] \leqslant k \mathcal{H}_{\lambda} w(x)
$$

By the monotonicity of $\varphi^{-1}(w)$, we obtain

$$
\varphi^{-1}(w(t)) \leqslant \mathcal{H}_{\lambda} \varphi^{-1}(w(t)),
$$

also we have

$$
w(t) \leqslant \varphi\left[\mathcal{H}_{\lambda} \varphi^{-1}(w(t))\right]
$$

Then by using (5.4), we see that

$$
\sup _{x>0} \frac{1}{\Lambda(x)} \int_{a}^{x} \lambda(t) \mathcal{H}_{\lambda} w(t) d t \leqslant k \frac{1}{\Lambda(x)} \int_{a}^{x} \lambda(t) w(t) d t \leqslant k \frac{1}{\Lambda(x)} \int_{a}^{x} \lambda(t) \varphi\left[\mathcal{H}_{\lambda} \varphi^{-1}(w(t))\right] d t .
$$

Then, we get

$$
\sup _{x>0} \mathcal{H}_{\lambda}\left[\mathcal{H}_{\lambda} w(t)\right] \leqslant k \mathcal{H}_{\lambda}\left[\varphi\left[\mathcal{H}_{\lambda} \varphi^{-1}(w(t))\right]\right]
$$

So we obtain

$$
\sup _{x>0} \mathcal{H}_{\lambda} w(x) \leqslant k \varphi\left[\mathcal{H}_{\lambda} \varphi^{-1}(w(x))\right]
$$

Then by applying the Jensen inequality for $\varphi^{-1}(w(t))$, and using (5.4), (5.5), so we have

$$
\begin{aligned}
\sup _{x>0} \mathcal{H}_{\lambda}\left[\varphi\left(\mathcal{H}_{\lambda} \varphi^{-1}(w(t))\right)\right] & =\sup _{x>0} \frac{1}{\Lambda(x)} \int_{0}^{x} \lambda(t) \varphi\left(\frac{1}{\Lambda(t)} \int_{0}^{t} \lambda(s) \varphi^{-1}(w(s)) d s\right) d t \\
& \leqslant \sup _{x>0} \mathcal{H}_{\lambda}\left[\mathcal{H}_{\lambda} w(t)\right] \leqslant k \mathcal{H}_{\lambda} w(x) \leqslant k^{2} \varphi\left[\mathcal{H}_{\lambda} \varphi^{-1}(w(x))\right] .
\end{aligned}
$$

Assume that $\mathcal{A}_{1, \lambda}\left[\varphi\left(\mathcal{H}_{\lambda} \varphi^{-1}(\mathcal{w}(\mathrm{t}))\right)\right]=\mathcal{C}$. Then

$$
\sup _{x>0} \mathcal{H}_{\lambda}\left[\varphi\left(\mathcal{H}_{\lambda} \varphi^{-1} \mathcal{w}(\mathrm{t})\right)\right] \leqslant \mathcal{C} \varphi\left[\mathcal{H}_{\lambda} \varphi^{-1}(\boldsymbol{w}(x))\right]
$$

By the monotonicity of $\varphi^{-1}(w)$, we get that

$$
\sup _{x>0} \mathcal{H}_{\lambda} w(x) \leqslant \sup _{x>0} \mathcal{H}_{\lambda}\left[\varphi\left(\mathcal{H}_{\lambda} \varphi^{-1}(w(t))\right)\right] \leqslant \mathcal{C} \varphi\left[\mathcal{H}_{\lambda} \varphi^{-1}(w(x))\right]
$$

By using (5.7), we obtain

$$
\sup _{x>0} \mathcal{H}_{\lambda}\left[\mathcal{H}_{\lambda} w(t)\right] \leqslant e \mathcal{H}_{\lambda}\left[\varphi\left(\mathcal{H}_{\lambda} \varphi^{-1}(w(t))\right)\right]
$$

and by applying the Jensen inequality for $\varphi^{-1}(w(x))$, we have that

$$
\varphi\left[\mathcal{H}_{\lambda} \varphi^{-1}(w(x))\right] \leqslant \mathcal{H}_{\lambda} w(x) .
$$

Then by using (5.8), (5.6), and (5.9), we get

$$
\sup _{x>0} \mathcal{H}_{\lambda}\left[\mathcal{H}_{\lambda} w(t)\right] \leqslant \mathcal{e} \mathcal{H}_{\lambda}\left[\varphi\left(\mathcal{H}_{\lambda} \varphi^{-1}(w(t))\right)\right] \leqslant \mathcal{C}^{2} \varphi\left[\mathcal{H}_{\lambda} \varphi^{-1}(w(x))\right] \leqslant \mathcal{C}^{2} \mathcal{H}_{\lambda} w(x),
$$

and so $w \in \mathcal{A}_{1, \lambda}$. The proof is complete. 


\section{Acknowledgment}

The authors are grateful to the editor and anonymous reviewers for their valuable and insightful comments.

\section{References}

[1] R. P. Agarwal, D. O'Regan, S. H. Saker, Hardy Type Inequalities on Time Scales, Springer, Cham, (2016). 1

[2] L. Basile, L. D'Apuzzo, M. Squillante, Relations between Gehring classesand Muckenhoupt classes via the Hardy operator, Ricerche di Mat., 45 (1996), 157-178. 2

[3] L. Basile, L. D’Apuzzo, M. Squillante, The limit class of Gehring type $\mathrm{G}_{\infty}$, Boll. Un. Mat. Ital. B (7), 11 (1997), 871-884. 1

[4] B. Bojarski, C. Sbordone, I. Wik, The Muckenhoupt class $\mathcal{A}_{1}(\delta)$, Studia Math., 101 (1992), 155-163. 1, 1

[5] R. R. Coifman, C. Fefferman, Weighted norm inequalities for maximal weights and singular integrals, Studia Math., 51 (1974), 241-250. 1

[6] R. Corporente, Weighted Integral Inequalities, BMO-Spaces and Applications, Dissertation thesis, Italy, (1991). 1

[7] D. Cruz-Uribe, C. J. Neugebauer, The structure of the reverse Hölder classes, Tran. Amer. Math. Soc., 347 (1995), 2941-2960.

[8] L. D’Apuzzo, C. Sbordone, Reverse Hölder inequalities: A sharp result, Rend. Mat. Appl. (7), 10 (1990), 357-366.

[9] V. D. Didenko, A. A. Korenovskii, Power means and the reverse Hölder inequality, Studia Math., 207 (2011), 85-95.

[10] V. D. Didenko, A. A. Korenovskii, The reverse Hölder inequality for power means, J. Math. Sci. (N.Y.), 183 (2012), 762-771.

[11] M. Dindoš, T. Wall, The sharp $\mathcal{A}^{p}$ constant for weights in a reverse Hölder class, Rev. Mat. Iberoam., 25 (2009), $559-594$.

[12] J. Garcia-Cuerva, J. L. Rubio de Francia, Weighted Norm Inequalities and Related Topics, North-Holland Publishing Co., Amsterdam, (1985). 1

[13] F. W. Gehring, The $\mathrm{L}^{\mathrm{p}}$-integrability of the partial derivatives of a quasiconformal mapping, Bull. Amer. Math. Soc., 79 (1973), 465-466. 1

[14] F. W. Gehring, The $\mathrm{L}^{\mathrm{P}}$-integrability of the partial derivatives of a quasi-conformal mapping, Acta Math., 130 (1973), 265-277. 1

[15] G. H. Hardy, J. E. Littlewood, G. Polya, Inequalities, Cambridge University Press, Cambridge, (1934). 1, 2, 2

[16] R. Hunt, B. Muckenhoupt, R. Wheeden, Weighted norm inequalities for the conjugate function and Hilbert transform, Trans. Amer. Math. Soc., 176 (1973), 227-251. 1

[17] M. M. Iddrisu, C. A. Okpoti, Applications of Taylor series for Carleman's inequality through Hardy inequality, Korean J. Math., 23 (2015), 655-664. 2

[18] R. Johnson, C. J. Neugebauer, Homeomorphisms preserving $\mathcal{A}_{\mathrm{p}}$, Rev. Mat. Iberoamericana, 3 (1987), $249-273.1$

[19] A. A. Korenovskii, The exact continuation of a reverse Hölder inequality and Muckenhoupt's conditions, Math. Notes, 52 (1992), 1192-1201.

[20] A. A. Korenovskii, V. V. Fomichev, Self-improvement of summability factors of functions satisfying the reverse Hölder inequality in limit cases, Ukrainian Math. J., 62 (2010), 552-563. 1

[21] N. Levinson, Generalizations of an inequality of Hardy, Duke Math. J., 31 (1964), 389-394. 5.2

[22] T. X. Li, N. Pintus, G. Viglialoro, Properties of solutions to porous medium problems with different sources and boundary conditions, Z. Angew. Math. Phys., 70 (2019), 18 pages. 1

[23] T. X. Li, G. Viglialoro, Analysis and explicit solvability of degenerate tensorial problems, Bound. Value Probl., 2018 (2018), 13 pages.

[24] N. A. Malaksiano, The exact inclusion of Gehring classes in Muckenhoupt classes, Mat. Zametki, 70 (2001), $742-750$.

[25] N. A. Malaksiano, The precise embeddings of one-dimensional Muckenhoupt classes in Gehring classes, Acta Sci. Math. (Szeged), 68 (2002), 237-248. 1

[26] B. Muckenhoupt, Hermite conjugate expansions, Trans. Amer. Math. Soc., 139 (1969), 243-260. 1

[27] B. Muckenhoupt, Mean convergence of Hermite and Laguerre series. II, Trans. Amer. Math. Soc., 147 (1970), $433-460$. 1

[28] B. Mucheknhoupt, Weighted norm inequalities for the Hardy maximal function, Trans. Amer. Math. Soc., 165 (1972), 207-226. 1, 1, 1, 1

[29] B. Muckenhoupt, E. M. Stein, Classical expansions and their relation to conjugate harmonic functions, Trans. Amer. Math. Soc., 118 (1965), 17-92. 1

[30] J. Murica, G. Viglialoro, A singular elliptic problem related to the membrane equilibrium equations, Int. J. Comput. Math., 90 (2013), 2185-2196. 1

[31] A. Popoli, Sharp integrability exponents and constants for Muckenhoupt and Gehring weights as solutions to a unique equation, Ann. Acad. Sci. Fenn. Math., 43 (2018), 785-805.

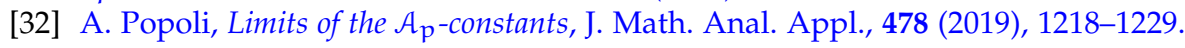

[33] S. H. Saker, D. O'Regan, R. P. Agarwal, A higher integrability theorem from a reverse weighted inequality, Bull. Lond. Math. Soc., 51 (2019), 967-977. 1 